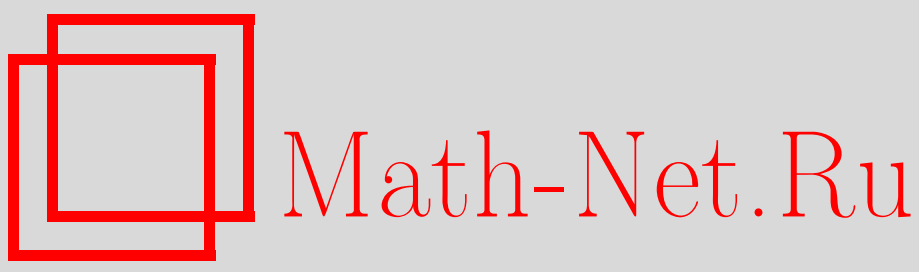

Д. В. Тураев, Л. П. Шильников, Пример дикого странного аттрактора, Матем. сб., 1998, том 189, номер 2, 137-160

DOI: https://doi.org/10.4213/sm300

Использование Общероссийского математического портала Math-Net.Ru подразумевает, что вы прочитали и согласны с пользовательским соглашением http://www . mathnet.ru/rus/agreement

Параметры загрузки:

IP: 3.82 .47 .9

26 апреля 2023 г., $16: 44: 46$ 
УДК 517.95

\author{
Д.В. Тураев, Л.П. Шильников
}

\title{
Пример дикого странного аттрактора
}

\begin{abstract}
В пространстве $C^{r}$-гладких, $r \geqslant 4$, потоков в $\mathbb{R}^{n}, n \geqslant 4$, доказьвается существование областей, заполненных системами, каждая из которых имеет аттрактор (здесь - вполне устойчивое цепно-транзитивное замкнутое инвариантное множество), содержащий нетривиальное базисное гиперболическое множество вместе с его неустойчивьм многообразием, которое при этом имеет точки нетрансверсального пересечения с устойчивьм многообразием. Приведена конструкция такого дикого аттрактора, содержащего состояние равновесия типа седло-фокус

Библиографияя: 22 названия.
\end{abstract}

\section{§1. Постановка задачи и основные результаты}

В настоящей работе мы выделяем класс динамических систем со странным аттрактором нового типа, чья особенность состоит в том, что он может содержать дикое гиперболическое множество (в смысле [1]). Именно, мы указьваем области в пространстве $C^{r}$-гладких, $r \geqslant 4$, потоков в $\mathbb{R}^{n}, n \geqslant 4$, заполненные системами, каждая из которых имеет аттрактор (здесь - вполне устойчивое цепно-транзитивное замкнутое инвариантное множество), содержащий нетривиальное базисное гиперболическое множество вместе с его неустойчивым многообразием, которое при этом имеет точки нетрансверсального пересечения с устойчивым многообразием.

Как показано в [1]-[5], наличие дикого множества обуславливает весьма нетривиальное поведение траекторий. Мы, в частности, показываем, что построенный нами аттрактор может содержать периодические траектории с негрубыми гомоклиническими кривыми, причем порядок касания устойчивого и неустойчивого многообразия вдоль гомоклинической кривой может быть произвольно велик. Так как описание бифуркаций касания порядка $n$ требует $n$ независимых параметров, данный результат означает, в частности, что полное описание динамики и бифуркаций построенного аттрактора невозможно ни в каком конечно-параметрическом семействе. Другим отражением этого свойства служит то, что данный аттрактор может содержать негрубые периодические траектории произвольно высоких порядков вырождения. Кроме того, в нем могут одновременно существовать периодические траектории с различными размерностями неустойчивых многообразий $\left(\operatorname{dim} W^{u}=2\right.$ и $\left.\operatorname{dim} W^{u}=3\right)$.

Наша конструкция близка к построенной в [6], [7] геометрической модели аттрактора Лоренца с той разницей, что она существенно нетрехмерна и что здесь состояние равновесия не седло, а седло-фокус. Именно, пусть $X-C^{r}$-гладкий, $r \geqslant 4$,

Работа вьполнена при частичной поддержке Российского фонда фундаментальных исследований (грант № 96-01-01135) и INTAS (грант № 930570). 
поток в $\mathbb{R}^{n}, n \geqslant 4$, имеющий состояние равновесия $O$ типа седло-фокус с характеристическими показателями $\gamma,-\lambda \pm i \omega,-\alpha_{1}, \ldots,-\alpha_{n-3}$, где $\gamma>0,0<\lambda<\operatorname{Re} \alpha_{j}$, $\omega \neq 0$. Будем предполагать, что

$$
\gamma>2 \lambda
$$

Данное условие введено в [8], где показана, в частности, его необходимость для того, чтобы в случае, когда сепаратриса возвращается в седло-фокус при $t \rightarrow+\infty$ (образует гомоклиническую петлю), в окрестностипетли не могли бы рождаться устойчивые периодические траектории.

Введем координаты $(x, y, z)\left(x \in \mathbb{R}^{1}, y \in \mathbb{R}^{2}, z \in \mathbb{R}^{n-3}\right)$ так, чтобы состояние равновесия находилось в начале координат, одномерное неустойчивое многообразие точки $O$ касалось оси $x$, а $(n-1)$-мерное устойчивое многообразие касалось плоскости $\{x=0\}$; при этом координаты $y_{1,2}$ отвечают ведущим показателям $\lambda \pm i \omega$, а координаты $z$ - неведущим показателям $\alpha$.

Предположим, что поток имеет секущую, скажем, поверхность П: $\{\|y\|=1$, $\|z\| \leqslant 1,|x| \leqslant 1\}$. Поскольку устойчивое многообразие $W^{s}$ касается в точке $O$ плоскости $\{x=0\}$, то локально оно задается уравнением вида $x=h^{s}(y, z)$, где $h^{s}$ - некоторая гладкая функция, $h^{s}(0,0)=0$. Мы предположим, что такое представление справедливо по крайней мере при $(\|y\| \leqslant 1,\|z\| \leqslant 1)$ и что при этом $\left|h^{s}\right|<1$. Таким образом, поверхность П служит секущей для $W_{\mathrm{loc}}^{s}$, и при этом пересечение $W_{\text {loc }}^{s}$ с П имеет вид $\Pi_{0}: x=h_{0}(\varphi, z)$, где $\varphi$ - угловая координата, $y_{1}=\|y\| \cos \varphi, y_{2}=\|y\| \sin \varphi$, а $h_{0}-$ некоторая гладкая функция, $-1<h_{0}<1$. Заменой координат можно с делать $h_{0} \equiv 0$, и мы будем считать, что это вьполнено.

Мы предполагаем, что все траектории, стартующие на $П \backslash \Pi_{0}$, возвращаются на П, определяя тем самьм отображения Пуанкаре: $T_{+}: \Pi_{+} \rightarrow \Pi$ и $T_{-}: \Pi_{-} \rightarrow \Pi$, где $\Pi_{+}=\Pi \cap\{x>0\}$ и $\Pi_{-}=\Pi \cap\{x<0\}$. Очевидно, что если $P$ - точка на П с координатами $(x, \varphi, z)$, то

$$
\lim _{x \rightarrow-0} T_{-}(P)=P_{-}^{1}, \quad \lim _{x \rightarrow+0} T_{+}(P)=P_{+}^{1},
$$

где $P_{-}^{1}$ и $P_{+}^{1}$ - точки первого пересечения одномерных неустойчивых сепаратрис точки $O$ с П. Соответственно, мы можем доопределить отображения $T_{+}$и $T_{-}$по непрерывности, так что

$$
T_{-}\left(\Pi_{0}\right)=P_{-}^{1}, \quad T_{+}\left(\Pi_{0}\right)=P_{+}^{1}
$$

Очевидно, что область $\mathscr{D}$, заполненная траекториями, стартующими на П (плюс точка $O$ и ее сепаратрисы), является поглощающей областью для системы $X$ в том смысле, что траектории, стартующие на $\partial \mathscr{D}$, входят в $\mathscr{D}$ и остаются там при всех положительных значениях времени $t$. По построению $\mathscr{D}$ представляет собой цилиндр $\{\|y\| \leqslant 1,\|z\| \leqslant 1,|x| \leqslant 1\}$ с двумя подклеенными ручками, окружающими сепаратрисы (рис. 1).

Мы предположим, что (полу)поток в $\mathscr{D}$ псевдогиперболичен. (Нам удобнее здесь вкладывать в это понятие более сильный смысл, чем это делается обычно [9].) Именно, мы предлагаем следующее.

ОПРЕДЕЛЕНИЕ. Полупоток называется псевдогиперболическим, если выполнены два следуюших условия:

(А) в каждой точке фазового пространства касательное пространство инвариантным относительно линеаризованного полупотока образом раскладывается в прямую сумму подпространств $N_{1}$ и $N_{2}$, непрерывно зависящих от 


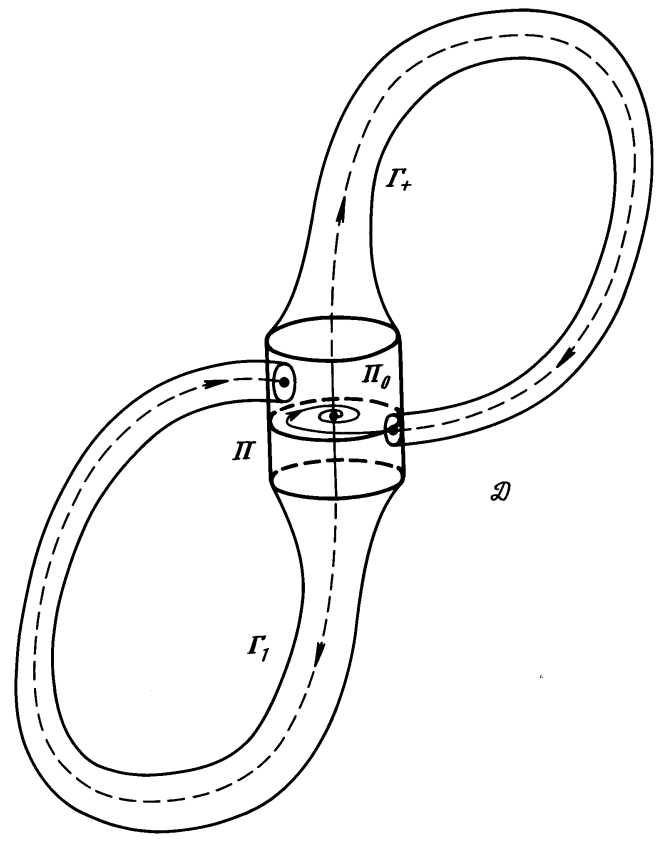

Рис. 1

точки, так что максимальньй ляпуновский показатель, отвечающий $N_{1}$, строго меньше минимального ляпуновского показателя, отвечающего $N_{2}$ : для любой точки $M$, для любых ненулевых векторов $u \in N_{1}(M)$ и $v \in N_{2}(M)$

$$
\limsup _{t \rightarrow+\infty} \frac{1}{t} \ln \frac{\left\|u_{t}\right\|}{\|u\|}<\liminf _{t \rightarrow+\infty} \frac{1}{t} \ln \frac{\left\|v_{t}\right\|}{\|v\|},
$$

где $u_{t}$ и $v_{t}-$ сдвиг векторов $u$ и $v$ полупотоком, линеаризованньпм вдоль траектории точки $M$;

(В) линеаризованный полупоток әкспоненциально растягивает объем в ограничении на $N_{2}$.

Новшество состоит здесь во введении условия (В), которое гарантирует отсутствие устойчивых периодических траекторий. Вообще говоря, данное определение не исключает случай, когда максимальньй ляпуновский показатель, отвечающий $N_{1}$, всюду неотрицателен - тогда линеаризованный полупоток тем более является растягивающим в ограничении на $N_{2}$, и условие (В) вьполнено тривиально. В данной работе мы, напротив, рассматриваем случай, когда линеаризованный полупоток является әкспоненциально сжимающим в ограничении на $N_{1}$, при этом условие (В) существенно.

Заметим, что псевдогиперболичность сохраняется при малых гладких возмушениях системы: согласно [9] инвариантное разложение касательного пространства сохраняется при малых возмушениях, и подпространства $N_{1}$ и $N_{2}$ меняются непрерывно. Очевидно, при этом, что свойство экспоненциального растяжения объемов в $N_{2}$ также устойчиво к малым возмушениям. 
Приведенное определение достаточно широко; оно включает, в частности, гиперболические потоки, для которых можно положить $\left(N_{1}, N_{2}\right)=\left(N^{s}, N^{u} \oplus N_{0}\right)$ или $\left(N_{1}, N_{2}\right)=\left(N^{s} \oplus N_{0}, N^{u}\right)$, где $N^{s}$ и $N^{u}$ - устойчивое и неустойчивое инвариантные подпространства, а $N_{0}$ - одномерное инвариантное подпространство, натянутое на вектор фазовой скорости. Геометрическая модель аттрактора Лоренца из [7] или [10] также относится к данному классу: здесь $N_{1}$ служит касательной к сжимающему инвариантному слоению коразмерности два, а растяжение площадей в двумерном подпространстве $N_{2}$ обеспечено тем, что отображение Пуанкаре является растягивающим в направлении, трансверсальном сжимающему слоению.

В данной работе мы предполагаем, что подпространство $N_{1}$ имеет коразмерность три: $\operatorname{dim} N_{1}=n-3, \operatorname{dim} N_{2}=3$, и что линеаризованный поток является при $t \geqslant 0$ экспоненциально сжимающим на $N_{1}$. Условие $(\mathrm{A})$ означает при этом, что для векторов из $N_{2}$ если и имеет место сжатие, то более слабое чем на $N_{1}$. Чтобы подчеркнуть последнее обстоятельство, мы будем называть $N_{1}$ сильно сжимающим, а $N_{2}$ - иентральным подпространством и обозначать их $N^{s s}$ и $N^{c}$, соответственно. Дополнительно мы будем считать, что координаты $(x, y, z)$ в $\mathbb{R}^{n}$ введены так, что в каждой точке области $\mathscr{D}$ пространство $N^{s s}$ имеет ненулевую проекцию на координатное пространство $z$, а $N^{c}$ - ненулевую проекцию на координатное пространство $(x, y)$.

Заметим, что требования псевдогиперболичности изначально выполнены в точке $O$ : здесь $N^{s s}$ совпадает с координатньм пространством $z$, а $N^{c}-$ с $(x, y)$, условие (1) как раз гарантирует растяжение объемов в инвариантном подпространстве $(x, y)$. Эти свойства линеаризованного потока автоматически наследуются траекториями в малой окрестности $O$. По существу, мы потребовали наследования этих свойств в немалой окрестности $\mathscr{D}$ точки $O$.

Согласно [9] экспоненциальное сжатие в $N^{s s}$ влечет существование инвариантного сжимающего слоения $\mathscr{N}^{s s}$ с $C^{r}$-гладкими слоями, касательными к $N^{s s}$. Аналогично [11], [12] можно показать, что это слоение абсолютно непрерывно. При факторизации по слоям область $\mathscr{D}$ становится ветвленым трехмерным многообразием: поскольку $\mathscr{D}$ ограничена, а фактор-полупоток растягивает объем, то очевидно, траектории фактор-полупотока должны склеиваться на некоторых поверхностях, чтобы оставаться ограниченными (ср. [10]).

Естественньм образом свойства псевдогиперболичности и растяжения объемов наследуются отображением Пуанкаре $T \equiv\left(T_{+}, T_{-}\right)$на секушей П. Здесь

$\left(\mathrm{A}^{*}\right)$ сушествует слоение из гладких слоев вида $(x, \varphi)=\left.h(z)\right|_{-1 \leqslant z \leqslant 1}$, где производная $h^{\prime}(z)$ равномерно ограничена, обладающее следуюшими свойствами: оно инвариантно в том смысле, что если $l$ - слой слоения, то $T_{+}^{-1}\left(l \cap T_{+}\left(\Pi_{+} \cup \Pi_{0}\right)\right)$ и $T_{-}^{-1}\left(l \cap T_{-}\left(\Pi_{-} \cup \Pi_{0}\right)\right)$ также являются слоями слоения (если только это не пустые множества), оно абсолютно непрерывно в том смысле, что отображение проектирования по слоям с одной трансверсали к слоению на другую изменяет площади в конечное число раз и при этом соответствующие отношения площадей отделены от бесконечности и нуля, оно сжимающее в том смысле, что если две точки принадлежат одному слою, то расстояние между их итерациями под действием отображения $T$ экспоненциально стремится к нулю;

$\left(\mathrm{B}^{*}\right)$ фактор-отображения $\widetilde{T}_{+}$и $\widetilde{T}_{-}$экспоненциально растягивают площадь. 
Выполнения свойств $\left(\mathrm{A}^{*}\right),\left(\mathrm{B}^{*}\right)$ и соотношения (2) для отображения Пуанкаре $T$ достаточно для справедливости теорем 1-3 ниже. Подобно [7], где аналогичньй результат доказан для аттрактора Лоренца, можно установить достаточные условия для вьполнения $\left(\mathrm{A}^{*}\right)$ и $\left(\mathrm{B}^{*}\right)$.

Лемма 1. Запишем отображсение $T$ в виде

$$
(\bar{x}, \bar{\varphi})=g(x, \varphi, z), \quad \bar{z}=f(x, \varphi, z),
$$

где $f$ и $g$ - функции, гладкие при $x \neq 0$, терпящие разрыв при $x=0$ :

$$
\lim _{x \rightarrow-0}(g, f)=\left(x_{-}, \varphi_{-}, z_{-}\right) \equiv P_{-}^{1}, \quad \lim _{x \rightarrow+0}(g, f)=\left(x_{+}, \varphi_{+}, z_{+}\right) \equiv P_{+}^{1} .
$$

Пусть

$$
\operatorname{det} \frac{\partial g}{\partial(x, \varphi)} \neq 0
$$

Обозначим

$$
\begin{aligned}
A & =\frac{\partial f}{\partial z}-\frac{\partial f}{\partial(x, \varphi)}\left(\frac{\partial g}{\partial(x, \varphi)}\right)^{-1} \frac{\partial g}{\partial z}, & B & =\frac{\partial f}{\partial(x, \varphi)}\left(\frac{\partial g}{\partial(x, \varphi)}\right)^{-1} \\
C & =\left(\frac{\partial g}{\partial(x, \varphi)}\right)^{-1} \frac{\partial g}{\partial z}, & D & =\left(\frac{\partial g}{\partial(x, \varphi)}\right)^{-1} .
\end{aligned}
$$

При условии, что

$$
\begin{gathered}
\lim _{x \rightarrow 0} C=0, \quad \lim _{x \rightarrow 0}\|A\|\|D\|=0, \\
\sup _{P \in \Pi \backslash \Pi_{0}} \sqrt{\|A\|\|D\|}+\sqrt{\sup _{P \in \Pi \backslash \Pi_{0}}\|B\| \sup _{P \in \Pi \backslash \Pi_{0}}\|C\|}<1,
\end{gathered}
$$

отображение имеет непрерывное инвариантное слоение из гладких слоев ви$\partial а(x, \varphi)=\left.h(z)\right|_{-1 \leqslant z \leqslant 1}$, где производная $h^{\prime}(z)$ равномерно ограничена. Если, кроме того,

$$
\sup _{P \in \Pi \backslash \Pi_{0}}\|A\|+\sqrt{\sup _{P \in \Pi \backslash \Pi_{0}}\|B\| \sup _{P \in \Pi \backslash \Pi_{0}}\|C\|}<1,
$$

то слоение сжимающее и при дополнительном условии, что для некоторого $\beta>0$

функции $A|x|^{-\beta}, D|x|^{\beta}, B, C$ равномерно ограниченьи и гёльдеровь, $\frac{\partial \ln \operatorname{det} D}{\partial z}, \frac{\partial \ln \operatorname{det} D}{\partial(x, \varphi)} D|x|^{\beta}$ равномерно ограничень,

абсолютно непрерывное. Если при этом

$$
\sup _{P \in \Pi \backslash \Pi_{0}} \sqrt{\operatorname{det} D}+\sqrt{\sup _{P \in \Pi \backslash \Pi_{0}}\|B\| \sup _{P \in \Pi \backslash \Pi_{0}}\|C\|}<1,
$$

то фактор-отображение $\widetilde{T}$ растягивает площадь. 
Заметим, что как следует из [8], в случае, когда состояние равновесия - седло-фокус, отображение Пуанкаре вблизи $\Pi_{0}=\Pi \cap W^{s}$ записывается при подходящем выборе координат в виде

$$
(\bar{x}, \bar{\varphi})=Q_{ \pm}(Y, Z), \quad \bar{z}=R_{ \pm}(Y, Z) .
$$

Здесь

$$
\begin{aligned}
Y & =|x|^{\rho}\left(\begin{array}{rr}
\cos (\Omega \ln |x|+\varphi) & \sin (\Omega \ln |x|+\varphi) \\
-\sin (\Omega \ln |x|+\varphi) & \cos (\Omega \ln |x|+\varphi)
\end{array}\right)+\Psi_{1}(x, \varphi, z), \\
Z & =\Psi_{2}(x, \varphi, z),
\end{aligned}
$$

где $\rho=\lambda / \gamma<\frac{1}{2}($ см. (1)), $\Omega=\omega / \gamma$ и для некоторого $\eta>\rho$

$$
\left\|\frac{\partial^{p+|q|} \Psi_{i}}{\partial x^{p} \partial(\varphi, z)^{q}}\right\|=O\left(|x|^{\eta-p}\right), \quad 0 \leqslant p+|q| \leqslant r-2 ;
$$

$Q_{ \pm}, R_{ \pm}$в $(9)$ ( “+" отвечает $x>0$ - отображение $T_{+}$, “-” отвечает $x<0$ отображение $\left.T_{-}\right)$- гладкие функции в окрестности $(Y, Z)=0$, для которых можно записать разложение в ряд Тейлора

$$
Q_{ \pm}=\left(x_{ \pm}, \varphi_{ \pm}\right)+a_{ \pm} Y+b_{ \pm} Z+\cdots, \quad R_{ \pm}=z_{ \pm}+c_{ \pm} Y+d_{ \pm} Z+\cdots .
$$

Из (9)-(12) видно, что если $O$ - седло-фокус, удовлетворяющий (1), то, если $a_{+} \neq 0$ и $a_{-} \neq 0$, отображение $T$ удовлетворяет условиям (4) и $(7)$ с $\beta \in(\rho, \eta)$. Кроме того, вьполнены аналоги условий $(3),(5),(6),(8)$, где супремум берется не по $|x| \leqslant 1$, а только по малым $x$. Несложно продолжить отображение $(9),(10),(12)$ на всю П, так чтобы условия леммы выполнялись полностью. Таково, например, отображение

$$
\begin{aligned}
& \bar{x}=0.9|x|^{\rho} \cos (\ln |x|+\varphi), \\
& \bar{\varphi}=3|x|^{\rho} \sin (\ln |x|+\varphi), \\
& \bar{z}=\left(0.5+0.1 z|x|^{\eta}\right) \operatorname{sign} x,
\end{aligned}
$$

где $0.4=\rho<\eta$.

Как уже говорилось, растяжение объемов фактор-полупотоком накладьвает ограничения на возможные типы предельного поведения траекторий. Именно, в $\mathscr{D}$ не мохсет бъть устойчивых периодических траекторий. Более того, любая траектория в $\mathscr{D}$ имеет положительный показатель Ляпунова. Следовательно, в данном случае мы должны говорить о странном аттракторе.

Предварительно, напомним ряд определений и простых фактов из топологической динамики (см., например, [13], [14] и указанную там литературу). Пусть $X_{t} P-$ сдвиг точки $P$ по траектории потока $X$ за время $t$. Для данных $\varepsilon>0$ и $\tau>0$ назовем $(\varepsilon, \tau)$-траекторией последовательность точек $P_{1}, P_{2}, \ldots, P_{k}$ таких, что $P_{i+1}$ лежит на расстоянии меньшем $\varepsilon$ от $X_{t} P_{i}$ для некоторого $t>\tau$. Точку $Q$ назовем $(\varepsilon, \tau)$-достижимой из точки $P$, если сушествует $(\varepsilon, \tau)$-траектория, соединяющая $P$ и $Q$, и достижимой из точки $P$, если при некотором $\tau>0$ она $(\varepsilon, \tau)$-достижима из $P$ для любого $\varepsilon$ (это определение, очевидно, не зависит от выбора $\tau$ ). Множество $C$ достижимо из точки $P$, если оно содержит точку, достижимую из $P$. Точка $P$ называется иепно-рекурентной, если она достижима из точки $X_{t} P$ при любом $t$. Замкнутое инвариантное множество $C$ называется иепно-транзитивнылм, если для любых точек $P$ и $Q$ из $C$ и для любых $\varepsilon>0$ и $\tau>0$ в $C$ лежит 
$(\varepsilon, \tau)$-траектория, соединяющая $P$ и $Q$. Очевидно, все точки цепно-транзитивного множества цепно-рекуррентны.

Компактное инвариантное множество $C$ называется орбитно устойчивым, если для любой его окрестности $U$ найдется окрестность $V(C) \subseteq U$ такая, что траектории, стартующие в $V$, не покидают $U$ при $t \geqslant 0$. Орбитно устойчивое множество назовем вполне устойчивым, если для любой окрестности $U(C)$ найдутся $\varepsilon_{0}>0, \tau>0$ и окрестность $V(C) \subseteq U$ такая, что $\left(\varepsilon_{0}, \tau\right)$-траектории, стартующие в $V$, не покидают $U$ (очевидно, что это не что иное, как устойчивость по отношению к постоянно действующим возмущениям). Известно, что множество $C$ орбитно-устойчиво тогда и только тогда, когда $C=\bigcap_{j=1}^{\infty} U_{j}$, где $U_{j}$ - система вложенных друг в друга инвариантных открытых множеств, и вполне устойчиво, когда множества $U_{j}$ не только инвариантны, но и являются поглощающими областями (т.е. траектории, стартующие на $\partial U_{j}$, входят внутрь $U_{j}$ за время, не превьшаюшее некоторого $\tau_{j}$; очевидно при этом, что $(\varepsilon, \tau)$-траектории, стартующие на $\partial U_{j}$, всегда остаются внутри $U_{j}$, если $\varepsilon$ достаточно мало и $\left.\tau \geqslant \tau_{j}\right)$. Поскольку максимальное инвариантное множество (максимальный аттрактор), которое содержится в каждой поглощающей области, очевидно, асимптотически устойчиво, то всякое вполне устойчивое множество либо асимптотически устойчиво, либо является пересечением счетного числа вложенных друг в друга замкнутых инвариантных асимптотически устойчивых множеств.

Возвращаясь к исходной системе $X$, назовем аттрактором системы множество $\mathscr{A}$ точек, достижимых из состояния равновесия $O$ (в частности, множество $\mathscr{A}$ содержит сепаратрисы точки $O$ и их замыкание). Определенный выбор множества $\mathscr{A}$ обусловлен тем, что как показано в следуюшем параграфе, множество $\mathscr{A}$ иепно-транзитивно, вполне устойчиво и достижимо из любой точки поглощающей области $\mathscr{D}$; более того, $\mathscr{A}$ - единственное в $\mathscr{D}$ иепно-транзитивное вполне устойчивое множество и оно является пересечением всех вполне устойчивых инвариантных подмножеств $\mathscr{D}$ (теоремы 1 и 2).

Эти утверждения вытекают в основном из того, что в $\mathscr{D}$ nлотны moчкu, acuмnтотические к $O$ при $t \rightarrow+\infty$ (т.е. устойчивое многообразие точки $O$ плотно в $\mathscr{D}$ ) (лемма 2), что в свою очередь следует из того, что отображение Пуанкаре $T$, факторизованное по слоям сжимаюшего слоения, растягивает плошади. Отметим, что аналогичное свойство имеет место и для аттрактора Лоренца. Также по аналогии с аттрактором Лоренца мы покажем (теорема 3 ), что число связных компонент пересечения аттрактора $\mathscr{A}$ с секущей ограниченно, и дадим оценку числа компонент, аналогичную оценке числа лакун в аттракторе Лоренца [7].

Эта оценка важна при доказательстве теоремы 4, где мы строим дикое множество, содержашееся в аттракторе $\mathscr{A}$. Данная теорема составляет основной результат работы.

Отметим, что дикое множество в теореме 4 указывается вполне конструктивно. Для этого мы вводим дополнительное предположение, что система $X$ имеет гомоклиническую петлю седло-фокуса $O$ (рис. 2). Точнее, мы рассматриваем однопараметрическое семейство $X_{\mu}$ описанного вида и предполагаем, что при $\mu=0$ имеется гомоклиническая петля, т.е. одна из сепаратрис возвращается в точку $O$ при $t \rightarrow+\infty$. Системы с гомоклинической петлей образуют в пространстве динамических систем бифуркационные поверхности коразмерности один, и не уменьшая общности, можно предполагать, что при $\mu=0$ семейство $X_{\mu}$ пересекает соответ- 
ствующую поверхность трансверсально. Теорема 4 утверждает, что $\kappa \mu=0$ накапливается последовательность интервалов $\Delta_{i}$ таких, что при $\mu \in \Delta_{i}$ аттрактор $\mathscr{A}_{\mu}$ содержит дикое множсество и при этом для любого $\mu^{*} \in \Delta_{i}$ для любой системь, близкой $\kappa X_{\mu^{*}}$ в $C^{r}$-топологии, ее аттрактор $\mathscr{A}$ такжсе содержит дикое множество.

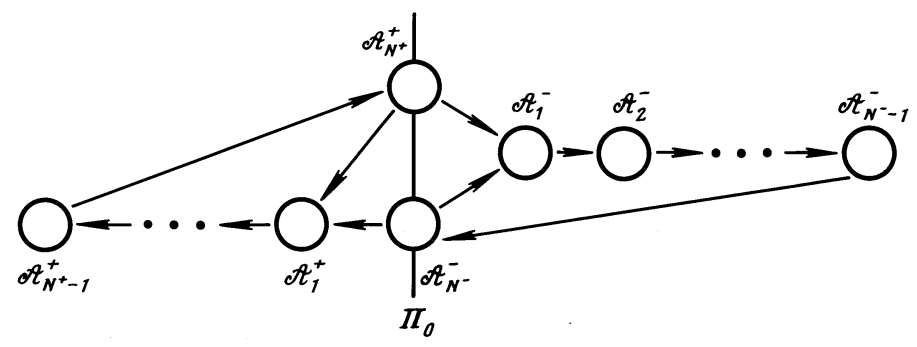

Рис. 2

Доказательство теоремы основано на следующих рассуждениях. Хорошо известно [15], что в окрестности гомоклинической петли седло-фокуса лежит нетривиальное транзитивное гиперболическое множество $\Lambda$. Это незамкнутое множество, в его замыкании лежит состояние равновесия $O$. При этом очевидно, что любая точка $\Lambda$ достижима из $O$, поэтому при $\mu=0$ множество $\Lambda$ лежит в аттракторе. Так как $\Lambda$ незамкнуто, оно не сохраняется целиком при малых изменениях $\mu$, однако его замкнутые инвариантые подмножества, конечно, сохраняются. Это базисные гиперболические множества, они неустойчивы и отделены от точки $O$. Тем не менее, мы явно выделяем подмножество, про которое удается доказать (леммы 3 и 4), что оно принадлежит аттрактору при всех малых $\mu>0$ (здесь положительные значения $\mu$ отвечают расщеплению петли вовнутрь) и для всех систем, близких к $X_{\mu}$ при $\mu>0$. Далее, в духе [8] мы показываем, что к $\mu=0$ накапливаются (с положительной стороны) значения $\mu=\mu_{i}$, отвечающие гомоклиническому касанию инвариантных многообразий периодической траектории из указанного подмножества (лемма 5). На основании [16] отсюда следует существование уже не отдельных значений $\mu$, а интервалов, на которых данное подмножество дикое (т.е. его неустойчивое многообразие при каждом $\mu$ из такого интервала имеет точки касания с устойчивым многообразием, и это свойство сохраняется при малых возмушениях системы), что завершает доказательство теоремы. Заметим, что применение результатов [16] требует проверки ряда условий невырожденности для гомоклинического касания при $\mu=\mu_{i}$, что сделано в лемме 5. Результаты, аналогичные [16], доказаны также в [17] при дополнительном предположении о существовании достаточно гладкой линеаризации для отображения Пуанкаре в окрестности периодической траектории, инвариантные многообразия которой имеют гомоклиническое касание. В нашем случае, однако, проверка этого условия была бы затруднительна и, возможно, потребовала бы дополнительных ограничений на спектр неведущих характеристических показателей $\alpha_{i}$ точки $O$, что не имеет отношения к сути дела.

Относительно общего случая, когда сепаратрисы точки $O$ не образуют петель, заметим, что как следует из плотности устойчивого многообразия в $\mathscr{D}$, сепаратрисы точки $O$ являются неблуждающими траекториями, поэтому естественно предположить, что их можно замкнуть мальм возмущением. Подобная проблема уже 
возникала в случае лоренцевского отображения и ее удалось преодолеть (в $C^{1}$-топологии), используя специфику отображения Пуанкаре. В настоящее время имеется весьма важная лемма Хаяши [18], которая также позволяет решить эту проблему. Поэтому и здесь справедливо

УТВЕРЖДЕНИЕ. Системы с гомоклинической петлей седло-фокуса О плотны в $C^{1}$-топологии в рассматриваемом классе систем.

Как уже отмечалось, наличие негрубых гомоклинических траекторий в аттракторе приводит к весьма нетривиальной динамике. Так, основываясь на результатах [4], [5], мы выводим из теоремы 4, что в построенных областях в пространстве динамических систем плотны системы, аттрактор которых содержит негрубые гомоклинические траектории произвольно высоких порядков касания (теорема 5), а также плотны системы, аттрактор которых содержит негрубъе периодические траектории произвольно высокого порядка вырожсдеия (теорема 6). В качестве частного случая теоремы 6 можно заметить, что в семействе $X_{\mu}$, в построенных в теореме 4 интервалах $\Delta_{i}$ плотны значения $\mu$, при которых аттрактор системы содержит периодическую траекторию типа седло-седло вместе с ее трехмерным неустойчивым многообразием, соответственно при данных значениях $\mu$ топологическая размерность аттрактора равна трем (теорема 7). Последнее, в частности, означает, что данный класс систем доставляет пример так называемого гиперхаоса.

\section{§2. Предварительное описание аттрактора псевдогиперболического потока}

В этом параграфе мы определим аттрактор системы $X$ и дадим оценку числа связных компонент его пересечения с секущей П (аналогичную оценке числа лакун в аттракторе Лоренца [7]).

ОПРЕДЕЛЕНИЕ. Назовем аттрактором системы множество $\mathscr{A}$ точек, достижимых из состояния равновесия $O$.

Это определение оправдано следующей теоремой.

Теорема 1. Мнохсество А् чепно-транзитивно, вполне устойчиво и достижимо из любой точки поглощающей области $\mathscr{D}$.

Устойчивость $\mathscr{A}$ следует непосредственно из определения: известно, что для любой исходной точки (и для точки $O$, в частности) множество точек, достижимых из нее, вполне устойчиво (систему поглощающих областей образуют множества точек, $\left(\varepsilon_{j}, \tau\right)$-достижимых из исходной точки, с произвольньми $\varepsilon_{j} \rightarrow+0$ и $\left.\tau>0\right)$.

Для доказательства оставшейся части теоремы заметим, что справедлива следующая

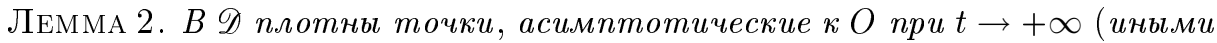
словами, устойчивое многообразие точки $O$ плотно в $\mathscr{D})$.

Действительно, возьмем произвольную точку на П и пусть $U$ - ее произвольная окрестность. Если бы $U$ не пересекалось с прообразами поверхности $\Pi_{0}=$ $W_{\text {loc }}^{s} \cap \Pi$, то при всех $i$ отображение $\left.T^{i}\right|_{U}$ было бы однозначно, и для множеств $T_{U}^{i}$ площади проекций на $z=0$ по слоям инвариантного слоения возрастали бы экспоненциально (в силу свойства (В)), что противоречит ограниченности П. Таким 
образом, прообразы $\Pi_{0}$ (а это - пересечения устойчивого многообразия точки $O$ с секушей П) плотны в П, а следовательно, устойчивое многообразие $O$ плотно в $\mathscr{D}$.

Из леммы 2 немедленно следует, что точка $O$ достижима из любой точки в $\mathscr{D}$. В частности, для любых точек $P$ и $Q$ из $\mathscr{A}$ точка $O$ достижима из $P$, в то же время, точка $Q$ достижима из $O$ по определению множества $\mathscr{A}$. Таким образом, $Q$ достижима из $P$ и для доказательства цепной транзитивности $\mathscr{A}$ осталось показать, что $(\varepsilon, \tau)$-траектории, соединяюшие $P$ и $Q$, можно выбирать лежашими в $\mathscr{A}$. Последнее, впрочем, следует из вполне устойчивости множества $\mathscr{A}$ : для любого $\delta>0$, как следует из определения, $(\varepsilon, \tau)$-траектория, соединяющая $P$ и $Q$, не покидает $\delta$-окрестности множества $\mathscr{A}$, если только $\varepsilon$ достаточно мало, и следовательно, эта траектория может быть аппроксимирована $\left(\varepsilon^{\prime}, \tau\right)$-траекторией, целиком лежашей в $\mathscr{A}$, где $\varepsilon^{\prime}$ может быть и больше чем $\varepsilon$, но очевидно, что $\varepsilon^{\prime} \rightarrow 0$ при $\varepsilon \rightarrow 0, \delta \rightarrow 0$.

Из теоремы 1 следует, что $\mathscr{A}$ - наименьшее вполне устойчивое множество в $\mathscr{D}$ : поскольку любое вполне устойчивое множество должно вместе с каждой своей точкой содержать все точки, достижимые из нее, то в силу теоремы 1 любое вполне устойчивое множество в $\mathscr{D}$ должно содержать точку $O$ и, вместе с ней, множество $\mathscr{A}$. Таким образом, $\mathscr{A}$ есть пересечение всех вполне устойчивых множеств в $\mathscr{D}$.

Следуюшая теорема показывает, что $\mathscr{A}$ - единственное цепно-транзитивное вполне устойчивое множество в $\mathscr{D}$.

Теорема 2. Любое орбитно устойчивое множество в $\mathscr{D}$, которое содержит точки, не принадлежащие $\mathscr{A}$, содержит не чепно-рекуррентнье точ$\kappa u$.

ДоказАТЕЛЬСТво. Поскольку устойчивое многообразие $O$ плотно в $\mathscr{D}$, то $O$, очевидно, принадлежит любому орбитно устойчивому подмножеству $\mathscr{D}$. Так как связная компонента орбитно устойчивого множества сама является орбитно устойчивым множеством, то каждая из них содержит точку $O$, и таким образом, любое орбитно устойчивое множество в $\mathscr{D}$ связно. Пусть $C$ - такое множество и пусть $P \in C, P \notin \mathscr{A}$. Так как $\mathscr{A}$ вполне устойчиво, то найдется поглошаюшая область $U$, содержащая $\mathscr{A}$ и не содержащая $P$. Так как точка $O$ принадлежит $\mathscr{A}$ и принадлежит $C$, то мы получили, что $C$ содержит как точки внутри $U$, так и точки вне $U$. Множество $C$ связно, следовательно, оно содержит хотя бы одну точку на $\partial U$, но как мы уже отмечали, $(\varepsilon, \tau)$-траектории, стартующие на $\partial U$, для достаточно малого $\varepsilon$ и достаточно большого $\tau$ всегда остаются внутри $U$ на конечном расстоянии от $\partial U$, т.е. ни одна точка на $\partial U$ не является цепно-рекуррентной.

Следуюшая теорема переносит на многомерный случай результат п. 1 теоремы 1.5 из [7] и дает описание разбиения пересечения аттрактора $\mathscr{A}$ с секущей П на компоненты связности - мы используем это при доказательстве теоремы 4. Обозначим через $q>1$ коэффициент растяжения плошадей фактор-отображением $(\widetilde{T})$ : если $V$ - область в П, не пересекаюшая $\Pi_{0}$, то

$$
S(T V)>q S(V),
$$

где через $S$ обозначена площадь проекции области на поверхность $\{z=0\}$ по слоям инвариантного слоения. Согласно $\left(\mathrm{B}^{*}\right) q>1$. Кроме того, так как $S(\Pi)=2 S\left(\Pi_{+}\right)$ и $T \Pi_{+} \subset \Pi$, то $S\left(T \Pi_{+}\right) / S\left(\Pi_{+}\right)<2$, и следовательно, мы имеем

$$
1<q<2 \text {. }
$$


Обозначим сепаратрисы точки $O$ через $\Gamma^{+}$и $\Gamma^{-}$, и пусть $\left\{P_{i}^{ \pm}\right\}$- точки последовательного пересечения $\Gamma^{ \pm}$с П. Эти последовательности могут быть бесконечны или конечны, последнее - если соответствующая сепаратриса образует петлю (возвращается в $O$ при $t \rightarrow+\infty)$.

Теорема 3. Число $N$ компонент связности множества $\mathscr{A} \cap \Pi$ конечно и справедлива оценка

$$
2 \leqslant N<2+\frac{|\ln (q-1)|}{\ln q} .
$$

Каждая компонента связности содержит по крайней мере одну из точек $P_{i}^{ \pm}$. При этом для некоторьх цельх $N_{+} \geqslant 1, N_{-} \geqslant 1, N_{+}+N_{-}=N$, удовлетворяющих неравенству

$$
q^{-N_{+}}+q^{-N_{-}}>1
$$

множество $\mathscr{A} \cap \Pi$ может бить представлено в виде

$$
\mathscr{A} \cap \Pi=\mathscr{A}_{1}^{+} \cup \cdots \cup \mathscr{A}_{N_{+}}^{+} \cup \mathscr{A}_{1}^{-} \cup \cdots \cup \mathscr{A}_{N_{-}}^{-},
$$

где $\mathscr{A}_{i}^{+}$и $\mathscr{A}_{i}^{-}$- компоненты, содерэсащие точки $P_{i}^{+}$и $P_{i}^{-}$, соответственно. $B$ әтой формуле все компоненты $\mathscr{A}_{i}^{ \pm}$различны,

$$
\begin{array}{ccc}
\mathscr{A}_{i}^{+} \cap \Pi_{0}=\varnothing \quad & \text { npu } i<N^{+}, & \mathscr{A}_{i}^{-} \cap \Pi_{0}=\varnothing \quad \text { npu } i<N^{-}, \\
\mathscr{A}_{N_{+}}^{+} \cap \Pi_{0} \neq \varnothing, & \mathscr{A}_{N_{-}}^{-} \cap \Pi_{0} \neq \varnothing,
\end{array}
$$

причем

$$
\begin{gathered}
T_{+}\left(\left(\mathscr{A}_{N_{-}}^{-} \cup \mathscr{A}_{N_{+}}^{+}\right) \cap\left(\Pi_{+} \cup \Pi_{0}\right)\right) \subseteq \mathscr{A}_{1}^{+}, \\
T_{-}\left(\left(\mathscr{A}_{N_{-}}^{-} \cup \mathscr{A}_{N_{+}}^{+}\right) \cap\left(\Pi_{-} \cup \Pi_{0}\right)\right) \subseteq \mathscr{A}_{1}^{-}, \\
\mathscr{A}_{i}^{-} \subseteq T^{i-1} \mathscr{A}_{1}^{-} \quad n p u \quad i<N^{-}, \quad \mathscr{A}_{i}^{+} \subseteq T^{i-1} \mathscr{A}_{1}^{+} \quad n p u \quad i<N^{+}
\end{gathered}
$$

(c.M. puc. 2).

ДокАЗАТЕЛЬСтво. Поскольку множество $\mathscr{A}$ орбитно устойчиво, то оно может быть представлено как пересечение последовательности вложенных друг в друга инвариантных областей. Соответственно, множество $\mathscr{A} \cap \Pi$ есть пересечение вложенных друг в друга окрестностей, инвариантных относительно положительных итераций отображения $T$. Пусть $U$ - одна из этих областей: $\mathscr{A} \cap \Pi \subset U, T U \subseteq U$. Мы можем удалить из $U$ компоненты связности, не содержашие точек из $\mathscr{A} \cap \Pi$, при этом $U$ останется инвариантным множеством. И так как $\mathscr{A} \cap \Pi$ компактно, то в $U$ останется только конечное число компонент. Пусть $V_{i}^{+}$и $V_{i}^{-}-$компоненты $U$, содержашие точки $P_{i}^{+}$и $P_{i}^{-}$, соответственно. Так как $P_{1}^{+} \in T_{+}\left(\Pi_{+} \cup \Pi_{0}\right)$ и $P_{1}^{-} \in T_{-}\left(\Pi_{-} \cup \Pi_{0}\right)$, а множества $T_{+}\left(\Pi_{+} \cup \Pi_{0}\right)$ и $T_{-}\left(\Pi_{-} \cup \Pi_{0}\right)$ отделены друг от друга, то $V_{1}^{+}$и $V_{1}^{-}$различны.

Заметим, что так как $T U \subseteq U$ и $T$ непрерывно на $\Pi \backslash \Pi_{0}$, то если какая-то компонента $U$ не пересекает $\Pi_{0}$, то ее образ лежит целиком внутри какой-либо другой компоненты (или совпадает с ней). Более точно, если какие-то точки $P_{1}$ и $P_{2}=T P_{1}$ принадлежат компонентам $V_{1}$ и $V_{2}$, соответственно, и если $V_{1} \cap \Pi_{0}=\varnothing$, то $T V_{1} \subseteq V_{2}$. Если же $V_{1}$ пересекает $\Pi_{0}$, то $V_{2}=V_{1}^{+}$или $V_{2}=V_{1}^{-}$, в зависимости 
от того, $P_{2}=T_{+} P_{1}$ или $P_{2}=T_{-} P_{1}$ (это следует из того, что $T_{+}\left(\Pi_{0}\right)=P_{1}^{+} \in V_{1}^{+}$ и $\left.T_{-}\left(\Pi_{0}\right)=P_{1}^{-} \in V_{1}^{-}\right)$. Таким образом, если $\left\{P_{j}\right\}-$ некоторая траектория $T$, a $V_{j}$ - компоненты $U$, содержашие $P_{j}$, то $T^{k} V_{j} \subseteq V_{j+k}, k \geqslant 1$, если только ни одна из компонент $V_{j}, \ldots, V_{j+k-1}$ не пересекает $\Pi_{0}$. Если же $V_{j} \cap \Pi_{0} \neq \varnothing$ при некотором $j$, то каждая компонента с номером, большим чем $j$, совпадает с одной из компонент $V_{i}^{ \pm}$.

Покажем теперь, что в $U$ нет других компонент связности кроме $V_{i}^{ \pm}$. Предположим, что некоторая компонента $V$ не содержит точек $P_{i}^{ \pm}$. По определению $V$ содержит хотя бы одну точку $P$ из $\mathscr{A}$. Так как множество $\mathscr{A} \cap \Pi$ инвариантно, т.е. состоит из целых траекторий, то оно содержит обратную полутраекторию $P_{0} \equiv P, P_{-1}, P_{-2}, \ldots\left(T P_{-(j+1)}=P_{-j}\right)$ точки $P$. Пусть $V_{0} \equiv V, V_{-1}, V_{-2}, \ldots-$ последовательность компонент связности, содержаших точки $P_{-j}$. Так как $V$ не совпадает по предположению ни с одной из компонент $V_{i}^{ \pm}$, то $V_{j}$ не пересекают $\Pi_{0}$, и $T V_{-(j+1)} \subseteq V_{-j}, j=0, \ldots, \infty$. Так как в $U$ только конечное число компонент, то последовательность $V_{-j}$ должна быть периодической, т.е. $V=V_{-k}$ для некоторого $k>0$. Это означало бы, что $T^{k}$ непрерьвно на $V$ и $T^{k} V \subseteq V$, что невозможно, так как при этом в силу растяжения площадей фактор-отображением должно быть $S(V)>q^{k} S(V)$ (см. (14)). Таким образом, множество $U$ исчерпывается компонентами $V_{i}^{ \pm}$.

Так как $T P_{i-1}^{+}=P_{i}^{+}$и $T P_{i-1}^{-}=P_{i}^{-}$, то $T V_{i-1}^{ \pm} \subseteq V_{i}^{ \pm}$до тех пор, пока $V_{i-1}^{ \pm} \cap \Pi_{0}=\varnothing$. Как мы отмечали (лемма 2), прообразы линии П 0 плотны в П. В частности, они пересекают $V_{1}^{+}$и $V_{1}^{-}$. Это означает, что некоторые итерации областей $V_{1}^{+}$и $V_{1}^{-}$пересекают $\Pi_{0}$. Следовательно, найдутся такие целые $N_{+}(U) \geqslant 1$ и $N_{-}(U) \geqslant 1$, что $V_{N_{+}(U)}^{+}$и $V_{N_{-}(U)}^{-}$пересекают $\Pi_{0}$, а компоненты $V_{i}^{ \pm}$с меншшими номерами не пересекают $\Pi_{0}$. Таким образом,

$$
\begin{array}{cl}
V_{i}^{+} \cap \Pi_{0}=\varnothing \text { при } i<N^{+}(U), & V_{i}^{-} \cap \Pi_{0}=\varnothing \text { при } i<N^{-}(U), \\
V_{N_{+}(U)}^{+} \cap \Pi_{0} \neq \varnothing, & V_{N_{-}(U)}^{-} \cap \Pi_{0} \neq \varnothing,
\end{array}
$$

причем

$$
\begin{gathered}
T_{+}\left(\left(V_{N_{-}(U)}^{-} \cup V_{N_{+}(U)}^{+}\right) \cap\left(\Pi_{+} \cup \Pi_{0}\right)\right) \subseteq V_{1}^{+}, \\
T_{-}\left(\left(V_{N_{-}(U)}^{-} \cup V_{N_{+}(U)}^{+}\right) \cap\left(\Pi_{-} \cup \Pi_{0}\right)\right) \subseteq V_{1}^{-}, \\
V_{i}^{-} \subseteq T^{i-1} V_{1}^{-} \text {при } i<N^{-}(U), \quad V_{i}^{+} \subseteq T^{i-1} V_{1}^{+} \text {при } i<N^{+}(U) .
\end{gathered}
$$

Из (21) следует, что

$$
U=V_{1}^{+} \cup \cdots \cup V_{N_{+}(U)}^{+} \cup V_{1}^{-} \cup \cdots \cup V_{N_{-}(U)}^{-} .
$$

Покажем, что $N_{+}(U)$ и $N_{-}(U)$ удовлетворяют неравенству

$$
q^{-N_{+}(U)}+q^{-N_{-}(U)}>1 .
$$

Действительно, очевидно, что

$$
\begin{aligned}
& S\left(\left(V_{N_{-}(U)}^{-} \cup V_{N_{+}(U)}^{+}\right) \cap \Pi_{+}\right) \geqslant S^{+} \equiv \max \left\{S\left(V_{N_{-}(U)}^{-} \cap \Pi_{+}\right), S\left(V_{N_{+}(U)}^{+} \cap \Pi_{+}\right)\right\}, \\
& S\left(\left(V_{N_{-}(U)}^{-} \cup V_{N_{+}(U)}^{+}\right) \cap \Pi_{-}\right) \geqslant S^{-} \equiv \max \left\{S\left(V_{N_{-}(U)}^{-} \cap \Pi_{-}\right), S\left(V_{N_{+}(U)}^{+} \cap \Pi_{-}\right)\right\}
\end{aligned}
$$


(напомним, что через $S$ обозначена плошадь проекции на поверхность $\{z=0\}$ по слоям инвариантного слоения). Отсюда и из $(21),(14)$ следует, что

$$
S\left(V_{N_{-}(U)}^{-}\right)>q^{N_{-}(U)} S^{-}, \quad S\left(V_{N_{+}(U)}^{+}\right)>q^{N_{+}(U)} S^{+} .
$$

Так как $S\left(V_{N_{-}(U)}^{-}\right) \leqslant S^{-}+S^{+}$и $S\left(V_{N_{+}(U)}^{-}\right) \leqslant S^{-}+S^{+}$, то получаем

$$
q^{-N_{-}(U)}>\frac{S^{-}}{S^{-}+S^{+}}, \quad q^{-N_{+}(U)}>\frac{S^{+}}{S^{-}+S^{+}},
$$

откуда следует (23).

Из (23) очевидно вытекает, что число компонент связности множества $U$ конечно и ограничено сверху константой, не зависящей от $U$ :

$$
N_{+}(U)+N_{-}(U)<2+\frac{|\ln (q-1)|}{\ln q} .
$$

Так как окрестность $U$ сколь угодно близка к $\mathscr{A} \cap \Pi$, то отсюда следует, что число компонент связности $\mathscr{A} \cap \Pi$ также конечно. Соответственно, если $U$ достаточно близка к $\mathscr{A} \cap \Pi$, то каждая компонента $U$ содержит ровно одну компоненту $\mathscr{A} \cap \Pi$. Теперь, из (20)-(24) следует (15)-(18) и, кроме того,

$$
\begin{gathered}
T_{+}\left(\left(\mathscr{A}_{N_{-}}^{-} \cup \mathscr{A}_{N_{+}}^{+}\right) \cap\left(\Pi_{+} \cup \Pi_{0}\right)\right) \subseteq \mathscr{A}_{1}^{+}, \\
T_{-}\left(\left(\mathscr{A}_{N_{-}}^{-} \cup \mathscr{A}_{N_{+}}^{+}\right) \cap\left(\Pi_{-} \cup \Pi_{0}\right)\right) \subseteq \mathscr{A}_{1}^{-}, \\
\mathscr{A}_{i}^{-} \subseteq T^{i-1} \mathscr{A}_{1}^{-} \text {при } i<N^{-}, \quad \mathscr{A}_{i}^{+} \subseteq T^{i-1} \mathscr{A}_{1}^{+} \text {при } i<N^{+} .
\end{gathered}
$$

Множество $\mathscr{A} \cap \Pi$ инвариантно относительно $T: T \mathscr{A} \cap \Pi=\mathscr{A} \cap \Pi$. Отсюда и из того, что $T$ является гомеоморфизмом множества $\Pi \backslash \Pi_{0}$ на его образ $T_{+} \Pi_{+} \cup T_{-} \Pi_{-}$, следует, что образ любой компоненты связности из $\mathscr{A} \cap \Pi$, не пересекающей $\Pi_{0}$, также является компонентой связности из $\mathscr{A} \cap$ П. Для компонент, пересекающих $\Pi_{0}$, объединение образов их пересечений с $\Pi_{+} \cup \Pi_{0}$ составляет $\mathscr{A}_{1}^{+}$, а объединение образов их пересечений с $\Pi_{-} \cup \Pi_{0}$ составляет $\mathscr{A}_{1}^{-}$. Таким образом, в $(25)$ можно заменить знак включения на равенство, что дает (19). Теперь, так как $\mathscr{A}_{1}^{+}$и $\mathscr{A}_{1}^{-}$различны, мы получаем, что все компоненты в (17) различны, что завершает доказательство теоремы.

Из доказательства видно, что теорема остается верна для произвольного инвариантного орбитно устойчивого множества в $\mathscr{D}$. Заметим также, что при доказательстве теорем 1-3 мы никак не использовали тот факт, что состояние равновесия $O$ - седло-фокус, т.е. они останутся верными и при условии, что $O$ - седло, лишш бы сушествовало инвариантное слоение и фактор-отображение растягивало площади. При этом несущественно, что слоение имеет коразмерность два: теоремы 1-3 останутся верны и в обшем случае, когда отображение $T$ имеет инвариантное абсолютно непрерывное сжимаюшее слоение коразмерности $k \geqslant 1$ и фактор-отображение растягивает $k$-мерные объемы. В эту схему, таким образом, укладывается и аттрактор Лоренца ( $k=1$ и $O$ - седло). 


\section{§3. Построение дикого аттрактора}

В этом параграфе мы докажем основной результат данной работы. Мы вернемся к случаю, когда $O$ - седло-фокус и сжимающее слоение имеет коразмерность два, покажем, что в этом случае аттрактор $\mathscr{A}$ может содержать дикое гиперболическое множество. В частности, это означает, что он отличен от аттрактора Лоренца (и от других известных аттракторов).

Возьмем однопараметрическое семейство $X_{\mu}$ систем рассматриваемого вида и предположим, что при $\mu=0$ имеется гомоклиническая петля седло-фокуса $О$, т.е. одна из сепаратрис (скажем, $\Gamma_{+}$) возвращается в точку $O$ при $t \rightarrow+\infty$ (рис. 2 ). Другими словами, мы предполагаем, что при $\mu=0$ семейство $X_{\mu}$ пересекает в пространстве динамических систем бифуркационную поверхность систем с гомоклинической петлей седло-фокуса, и мы будем считать, что данное пересечение трансверсально. Трансверсальность означает, что при изменении $\mu$ петля расщепляется и при этом, если $M$ - номер последней точки пересечения сепаратрисы $\Gamma_{+}$с секушей при $\mu=0\left(P_{M}^{+} \in \Pi_{0}\right.$ при $\left.\mu=0\right)$, то расстояние от точки $P_{M}^{+}$до $\Pi_{0}$ меняется "с ненулевой скоростью” при изменении $\mu$. Мы выберем знак $\mu$ так, чтобы $P_{M}^{+} \in \Pi_{+}$при $\mu>0$ (соответственно, $P_{M}^{+} \in \Pi_{-}$при $\left.\mu<0\right)$.

ТеОрема 4. $K \mu=0$ накапливается последовательность интервалов $\Delta_{i}$ таких, что при $\mu \in \Delta_{i}$ аттрактор $\mathscr{A}_{\mu}$ содержст дикое множество (нетривиальное транзитивное замкнутое гиперболическое множество, неустойчивое многообразие которого имеет точки касания с устойчивым многообразием). При этом для любого $\mu^{*} \in \Delta_{i}$ для любой системы, близкой $\kappa X_{\mu *}$ в $C^{r}$-топологии, ее аттрактор $\mathscr{A}$ также содержит дикое множсество.

ДокАЗАТЕЛЬСТво. По условию сепаратриса $\Gamma_{+}$при $\mu=0$ пересекает П в конечном числе точек $P_{i}^{+}$и последняя точка $P_{M}^{+}$принадлежит $\Pi_{0}$. Не уменьшая обшности, можно считать, что число $M$ достаточно велико. Именно, для всех малых $\mu$ фактор-отображение $\widetilde{T}$ растягивает площади, при этом очевидно, что коэффициент растяжения $q$ оценивается снизу константой, не зависящей от $\mu$. Соответственно, согласно теореме 3 число компонент связности аттрактора в пересечении с секущей П оценивается сверху константой $N$, не зависяшей от $\mu$, и мы будем считать ниже, что число $M$ точек пересечения не меньше $N+1$. Данное условие не является ограничением, поскольку согласно [19] гомоклиническую петлю седло-фокуса можно расшепить так, чтобы получить петлю сколь угодно высокой обходности по отношению к исходной. Более точно, к бифуркационной поверхности, отвечающей гомоклинической петле седло-фокуса, накапливаются поверхности, отвечающие гомоклиническим петлям сколь угодно высокой обходности. Семейство $X_{\mu}$, трансверсальное поверхности, отвечающей исходной петле, остается трансверсальньм и ко всем $C^{1}$-близким поверхностям. Соответственно, если при $\mu=0$ число $M$ точек пересечения петли $\Gamma_{+}$с П меньше $N+1$, то сколь угодно малым изменением $\mu$ можно получить петлю, у которой $M \geqslant N+1$ (и семейство $X_{\mu}$ останется трансверсальным соответствующей бифуркационной поверхности). Поэтому, доказав теорему для таких петель, мы автоматически получим утверждение теоремы и в общем случае.

Как известно [15], в любой малой окрестности гомоклинической петли седло-фокуса имеется счетное число однообходных (т.е. гомотопных петле в данной окрестности) седловых периодических траекторий (вообще говоря, это утвержде- 
ние имеет место только при выполнении некоторых дополнительных условий общего положения, которые, впрочем, в нашем случае выполнены автоматически, см. ниже). Каждая из этих траекторий пересекает малую секушую к петле в одной точке (мы возьмем в качестве такой секущей малую окрестность $\Pi_{0} \equiv W_{\mathrm{loc}}^{s} \cap \Pi$ в П). Данные траектории $L_{1}, L_{2}, \ldots$ можно занумеровать так, что точки пересечения $R_{1}, R_{2}, \ldots$ будут лежать от $W_{\text {loc }}^{s}$ на $\operatorname{paccтоянии~} \operatorname{dist}\left(R_{k}, W_{\mathrm{loc}}^{s}\right) \sim C e^{-\frac{\pi \gamma}{\omega} k}$, где $C$ - некоторая константа (напомним, что характеристические показатели седло- фокуса - это $\gamma,-\lambda \pm i \omega,-\alpha_{1}, \ldots,-\alpha_{n-3}$, где $\left.\gamma>0,0<\lambda<\operatorname{Re} \alpha_{j}, \omega \neq 0\right)$.

B [15] показано, что на секущей можно выделить накапливающиеся к пересечению секушей с $W_{\text {loc }}^{s}$ "полоски" - окрестности $\sigma_{1}, \sigma_{2}, \ldots$ точек $R_{1}, R_{2}, \ldots$, соответственно, такие, что при $\mu=0$ для любого $\rho^{\prime}>\rho=\lambda / \gamma$ сушествует $\bar{k}$ такое, что для любого $i \geqslant \bar{k}$ образ полоски $\sigma_{i}$ под действием отображения Пуанкаре (в нашем случае это отображение $T^{\prime} \equiv T^{M}$, где $M$ - число точек пересечения петли с П) правильно пересекает все полоски с номерами $j \geqslant \rho^{\prime} i$. "Правильно" означает здесь, что пересечение $T^{\prime} \sigma_{i} \cap \sigma_{j}$ связно и отображение $\left.T^{\prime}\right|_{\sigma_{i} \cap T^{\prime-1} \sigma_{j}}$ седловое в смысле [20]. Это означает [15], что в малой окрестности петли лежит гиперболическое инвариантное множество $\Lambda$, которое находится во взаимно-однозначном соответствии с множеством всевозможных бесконечных в обе стороны последовательностей целых чисел $\left\{j_{s}\right\}$, удовлетворяющих условию $j_{s} \geqslant \bar{k}, j_{s+1} \geqslant \rho^{\prime} j_{s}$ : последовательности $\left\{j_{s}\right\}$ отвечает траектория, последовательные точки пересечения которой с секущей лежат в полосках с номерами $\left\{j_{s}\right\}$. В частности, однообходной периодической траектории $L_{i}$ отвечает последовательность $\{\ldots$. iiiiii . . $\}$.

Надо отметить, что множество $\Lambda$ не замкнуто - его замьканию принадлежит состояние равновесия $O$, сепаратриса $\Gamma^{+}$и траектории в устойчивом многообразии. Для произвольного целого $k \geqslant \bar{k}$ выделим в $\Lambda$ замкнутое подмножество $\Lambda_{k}$, отвечающее последовательностям $\left\{j_{s}\right\}$, удовлетворяющим условию $j_{s} \leqslant k$. Так как при каждом фиксированном $k$ множество $\Lambda_{k}$ отделено от $W_{\text {loc }}^{s}$, то это замкнутое гиперболическое множество, следовательно, оно сохраняется при всех малых $\mu$ (и непрерывно зависит от $\mu$ ). Имеет место следующая

Лемма 3. Существует $C$ такое, что если $\bar{k}$ достаточно велико и $k \geqslant \bar{k}$, то мнохсество $\Lambda_{k}$ сохраняется при всех $\mu$ таких, что

$$
|\mu|<C e^{-\frac{\pi \rho \gamma}{\omega} k}
$$

Для упрошения записи мы обозначим через $\Lambda_{\mu}$ множество $\Lambda_{k}$, соответствующее наименьшему $k$, для которого выполнено (26). Лемма 3 доказывается на основе рассмотрений отображений вблизи гомоклинической петли седло-фокуса, не связанных со спецификой данной задачи. Следующий результат, напротив, сушественно использует теорему 3 и предположение, что гомоклиническая петля является достаточно многообходной.

ЛЕмма 4. При всех мальх $\mu \geqslant 0$ множсество $\Lambda_{\mu}$ принадлежсит аттракmopy $\mathscr{A}_{\mu}$.

Заметим, что при каждом фиксированном $\mu$ множество $\Lambda_{\mu}$ является замкнутым гиперболическим множеством, поэтому оно сохраняется и при малых возмущениях системы. Из доказательства леммы 4 станет видно, что и для всех достаточно близких систем это множество останется в аттракторе. 
Неустойчивое многообразие гиперболического множества $\Lambda_{\mu}$ также принадлежит аттрактору (в силу его $\varepsilon$-устойчивости). Мы докажем теорему, показав, что к $\mu=0$ накапливаются (с положительной стороны) интервалы значений $\mu$ (области Ньюхауса), при которых множество $\Lambda_{\mu}$ является диким, т.е. его неустойчивое многообразие касается устойчивого. Согласно [16] для этого достаточно показать, что к $\mu=0$ накапливается последовательность положительных значений $\mu$, при которых неустойчивое многообразие какой-либо периодической траектории из $\Lambda_{\mu}$ невырожденным образом касается ее устойчивого многообразия (мы уточним условия невырожденности ниже). Мы выберем в качестве такой периодической траектории однообходную траекторию $L_{j} \in \Lambda_{\mu}$. Искомый результат прямо вытекает из двух следуюших лемм.

Лемма 5. Существует последовательность $\mu_{j} \rightarrow+0$ такая, что при $\mu=\mu_{j}$ семейство $X_{\mu}$ трансверсально пересекает бифуркационную поверхность, на которой сепаратриса $\Gamma_{+}$ложится на устойчивое многообразие траектории $L_{j} \in \Lambda_{\mu}$, и при этом неустойчивое многообразие $L_{j}$ трансверсально пересекает $W^{s}(O)$.

Лемма 6. Сколь угодно близко $\kappa \mu=\mu_{j}$ имеются значения $\mu$, при которых устойчивое и неустойчивое многообразия седловой периодической траектории имеют невырожденное касание.

Перейдем к доказательству лемм 3-6. Доказательство лемм 3, 5, 6 проводится при помощи вычислений, аналогичных [8], [15], поэтому мы опустим детали и ограничимся только схемой. По условию при $\mu=0$ сепаратриса $\Gamma_{+}$образует гомоклиническую петлю: $P_{M}^{+}$(точка $M$-го пересечения $\Gamma_{+}$с П) лежит на $\Pi_{0}$. Заметим, что эта петля удовлетворяет условиям общего положения из [15]. Одно из этих условий состоит в том, что сепаратриса входит в $O$ (при $t \rightarrow+\infty$ ), касаясь ведушей плоскости, т.е. не лежит в сильно-устойчивом инвариантном многообразии точки $O$. Заметим, что сильно-устойчивое многообразие - это единственное $(n-3)$-мерное гладкое инвариантное многообразие, проходящее через $O$ и касательное в $O$ к собственному подпространству, отвечающему неведущим характеристическим показателям $-\alpha_{i}$. В нашем случае таким многообразием является слой слоения $\mathscr{N}^{s s}$, проходящий через $O$. Он имеет по предположению вид $(x, y)=h^{s s}(z)$ и не пересекает секушую $\Pi$, поэтому $\Gamma_{+}$не может лежать в нем. Другое условие обшего положения сформулировано в [15] как неравенство нулю некоторой величины и эквивалентно (см. [21]) наличию сильно-сжимающего слоения в окрестности петли - в нашем случае это условие вьполнено по предположению. Отметим, что, как следует из [21], [22], данные условия общего положения гарантируют, что система при всех малых $\mu$ имеет в окрестности петли инвариантное трехмерное $C^{1}$-многообразие $\mathscr{M}_{\mu}$, которое трансверсально слоению $\mathscr{N}^{s s}$ и которому принадлежат все траектории, остающиеся в малой окрестности петли при $t \rightarrow-\infty$.

Согласно [8] в подходящих координатах отображение Пуанкаре $\left.T^{\prime} \equiv T^{M}\right|_{\Pi_{+}}$ вблизи П 0 может быть записано в виде

$$
\begin{aligned}
& \bar{x}=x^{*}+a^{\prime} x^{\rho} \sin \left(\Omega \ln x+\varphi-\varphi^{*}\right)+\psi_{1}(x, \varphi, z, \mu), \\
& \bar{\varphi}=\varphi^{*}+b^{\prime} x^{\rho} \cos \left(\Omega \ln x+\varphi-\varphi^{*}+\theta\right)+\psi_{2}(x, \varphi, z, \mu), \\
& \bar{z}=z^{*}+\psi_{3}(x, \varphi, z, \mu),
\end{aligned}
$$


где $\left(x^{*}, \varphi^{*}, z^{*}\right)$ - координаты точки $P_{M}^{+}, a^{\prime}, b^{\prime}, \theta$ - некоторые величины и

$$
\left\|\frac{\partial^{p+|q|} \psi_{i}}{\partial x^{p} \partial(\varphi, z, \mu)^{q}}\right\|=O\left(|x|^{\eta-p}\right), \quad 0 \leqslant p+|q| \leqslant r-2,
$$

для некоторого $\eta>\rho$. Все константы в $(27)$ зависят от $\mu$. По условию $P_{M}^{+} \in \Pi_{0}$ (т.е. $x^{*}=0$ ) при $\mu=0$. Трансверсальность семейства $X_{\mu}$ бифуркационной поверхности, отвечающей гомоклиническим петлям означает, что $\left.\frac{\partial x^{*}}{\partial \mu}\right|_{\mu=0} \neq 0$. Не уменьшая общности, мы можем положить

$$
x^{*} \equiv \mu
$$

Отметим, что координаты, в которых справедливо представление (27), (28), вводятся так, что в ограничении на $W_{\text {loc }}^{s}$ производная $\dot{y}$ не зависит от $z$ (см. [8]). Поэтому слои инвариантного слоения $\mathscr{N}^{s s}$ в пересечении с $W_{\text {loc }}^{s}$ имеют вид $\{\varphi=$ const $\}$. Отсюда видно, что инвариантность слоения $\mathscr{N}^{s s} \cap$ П относительно отображения $\left(T^{\prime}\right)^{-1}$ эквивалентна условию

$$
a^{\prime} \neq 0, \quad b^{\prime} \neq 0, \quad \cos \theta \neq 0
$$

Неподвижные точки отображения $T^{\prime}$ находятся из условия $(\bar{x}=x, \bar{\varphi}=\varphi$, $\bar{z}=z)$. Следовательно, в этом случае из последних двух уравнений системы (27) можно при малых $x$ и $\mu$ выразить $\varphi$ и $z$ как функции $x$ и $\mu$ (см. (27)-(30)), после чего уравнение на координату $x$ примет вид

$$
x=\mu+a^{\prime} x^{\rho} \sin (\Omega \ln x)+o\left(x^{\rho}\right) .
$$

При $\mu=0$ для неподвижной точки $R_{j}$ имеем

$$
x_{j}=e^{-\frac{\pi j}{\Omega}}+o\left(e^{-\frac{\pi j}{\Omega}}\right)
$$

и

$$
\varphi_{j}=\varphi^{*}+o\left(e^{-\frac{\pi j}{\Omega}}\right), \quad z_{j}=z^{*}+o\left(e^{-\frac{\pi j}{\Omega}}\right),
$$

где $\left(x_{j}, \varphi_{j}, z_{j}\right)$ - координаты неподвижной точки, индекс $j$ пробегает все натуральные значения, начиная с некоторого достаточно большого $\bar{k}$. Очевидно, что соотношения $(31),(32)$ останутся справедливы и в случае $\mu \neq 0$, если только $\mu e^{\frac{\pi \rho j}{\Omega}}$ достаточно мало. Мультипликаторы неподвижной точки оцениваются следующим образом:

$$
\nu_{1} \sim(-1)^{j} a^{\prime} \Omega x_{j}^{\rho-1}, \quad \nu_{2} \sim-(-1)^{j} b^{\prime} \frac{\rho \cos \theta}{\Omega} x_{j}^{\rho}, \quad \nu_{m}=o\left(x_{j}^{\rho}\right), m=3, \ldots, n-1 .
$$

Отметим, что $\nu_{1}$ - единственный мультипликатор, больший единицы по абсолютной величине, а остальные лежат внутри единичного круга. Поэтому неустойчивое многообразие точки $R_{j}$ одномерно, а устойчивое $(n-2)$-мерно (соответственно, однообходная периодическая траектория $L_{j}$, которая пересекает П в точке $R_{j}$, имеет двумерное неустойчивое и $(n-1)$-мерное устойчивое многообразия). 
Чтобы найти устойчивое многообразие $W_{j}^{s}$ точки $R_{j}$, поступим следующим образом. Возьмем малый кусок поверхности $x=x_{j}$, содержащий точку $R_{j}$, и рассмотрим его прообраз относительно отображения $T^{-1}$. Согласно $(27)-(32)$ его можно представить в виде

$$
\sin \left(\Omega \ln x+\varphi-\varphi^{*}\right)=O\left(\frac{x_{j}}{x^{\rho}}+\frac{\mu}{x^{\rho}}+x^{\eta-\rho}\right)
$$

при условии, что $x$ достаточно мало. Мы будем считать, что

$$
x<2 e^{-\frac{\pi \bar{k}}{\Omega}}
$$

где $\bar{k}$ достаточно велико. При условии, что $x$ также и не слишком мало, именно, если малы величины $x_{j} / x^{\rho}$ и $\mu / x^{\rho}$, уравнение данной поверхности принимает вид

$$
\Omega \ln x=-\pi j-\varphi+\varphi^{*}+O\left(\frac{x_{j}}{x^{\rho}}+\frac{\mu}{x^{\rho}}+x^{\eta-\rho}\right)
$$

или

$$
x=e^{-\frac{\varphi-\varphi^{*}+\pi j}{\Omega}}(1+\cdots),
$$

где многоточием обозначена функция от $\varphi$ и $\mu$, малая вместе со своими производными до порядка $(r-2)$. Как мы отмечали, данное представление справедливо при условии, что $x, x_{j} / x^{\rho}$ и $\mu / x^{\rho}$ достаточно малы, т.е. на участке значений $\varphi$

$$
\pi \bar{k}<\varphi-\varphi^{*}+\pi j<\pi k
$$

где $\bar{k}$ - произвольное достаточно большое целое, не зависящее от $j$ и $\mu$, а $k$ может быть выбрано много большим $j$ при условии, что

$$
|\mu| e^{\frac{\pi \rho}{\Omega} k} \text { и } e^{\frac{\pi}{\Omega}(\rho k-j)} \text { малы }
$$

(последнее условие можно выполнить при $k$ много большем чем $j$, поскольку $\rho<1$ ). Пользуясь формулами (27)-(32), можно проверить, что прообраз относительно отображения $T^{-1}$ малого куска этой поверхности, содержашего неподвижную точку $R_{j}$, представляется в том же самом виде на том же самом интервале значений $\varphi$. То же самое остается справедливым для всех последующих итераций, причем величина $o(1)$ в (34) остается равномерно малой. Так как многообразие $W_{j}^{s}$ есть предел последовательности таких итераций, то получаем в результате, что на участке $(35),(36)$ устойчивое многообразие точки $R_{j}$ задается уравнением (34).

Для неустойчивого многообразия $W_{j}^{u}$ точки $R_{j}$ можно показать (взяв образ малого куска прямой $\varphi=\varphi_{j}$ и оценив поправку при последующих итерациях), что оно $C^{r-2}$-близко к спирали, задаваемой параметрическим соотношением вида

$$
\begin{aligned}
x & =\mu-a^{\prime} e^{-\frac{\rho}{\Omega} u} \sin u, \\
\varphi & =\varphi^{*}+b^{\prime} e^{-\frac{\rho}{\Omega} u} \cos (-u+\theta), \\
z & =z^{*}
\end{aligned}
$$


на участке, отвечаюшем значениям параметра $u>\pi \rho^{\prime} j$, где $\rho^{\prime}>\rho$ (и может быть выбрано сколь угодно близко к $\rho$ ), при условии, что $j$ достаточно велико и $|\mu| e^{\frac{\rho}{\Omega} j}$ мало. Отметим, что спираль наматывается на точку $P_{M}^{+}$и пересекает трансверсально П 0 . Из формул (34)-(37) видно, что $W_{j}^{u}$ имеет точки трансверсального пересечения с $W_{i}^{s}$ для $i>\rho^{\prime} j$. В частности, имеются точки трансверсального пересечения $W_{j}^{u}$ с $W_{j}^{s}$, и значит, точки $R_{j}$ включаются в нетривиальное гиперболическое множество.

Выберем малое $\xi>0$ и рассмотрим окрестности

$$
\sigma_{j}: e^{\xi}<x e^{\frac{\pi j}{\Omega}} e^{\frac{\varphi-\varphi^{*}}{\Omega}} e^{\frac{\operatorname{arctg}(\Omega / \rho)}{\Omega}}<e^{\pi-\xi},\left|\varphi-\varphi^{*}\right|<\pi
$$

точек $R_{j}$ (неформально говоря, мы исключили из рассмотрения значения $x$, отвечаюшие экстремумам функции $\left.x^{\rho} \sin (\Omega \ln x)\right)$. Пользуясь формулами $(27),(28)$, можно проверить, что при $\mu=0$ для любого $\rho^{\prime}>\rho$ сушествует $\bar{k}$ такое, что для любого $j \geqslant \bar{k}$ образ полоски $\sigma_{j}$ под действием отображения $T^{\prime}$ правильно пересекает все полоски с номерами $i \geqslant \rho^{\prime} j$ в том смысле, что пересечение $T^{\prime} \sigma_{j} \cap \sigma_{i}$ связно и отображение $\left.T^{\prime}\right|_{\sigma_{j} \cap T^{\prime-1} \sigma_{i}}$ седловое в смысле [20] (сильно растягивает по координате $x$ и сильно сжимает по координатам $(\varphi, z))$.

Пусть $\left\{P_{s}\right\}_{s=-\infty}^{s=+\infty}-$ траектория отображения $T^{\prime}$, целиком лежащая в объединении полосок $\bigcup_{j=\bar{k}}^{\infty} \sigma_{j}: P_{s} \in \sigma_{j_{s}}$. Назовем последовательность $\left\{j_{s}\right\}_{s=-\infty}^{s=+\infty} \kappa о д u-$ ровкой данной траектории. По лемме о последовательности седловых отображений [20] каждой кодировке, удовлетворяющей условию $j_{s} \geqslant \bar{k}, j_{s+1} \geqslant \rho^{\prime} j_{s}$, отвечает одна и только одна траектория с данной кодировкой. Объединение всех таких траекторий образует гиперболическое инвариантное множество $\Lambda$, которое содержит, в частности, все точки $R_{j}$. Несложно проверить, что при $\mu \neq 0$ образ полоски $\sigma_{j}$ по-прежнему правильно пересекает полоски $\sigma_{i}$ с $i>\rho^{\prime} j$, если только величина $|\mu| e^{\frac{\rho}{\Omega} j}$ достаточно мала, откуда в силу леммы о последовательности седловых отображений [20] следует утверждение леммы 3.

Для доказательства леммы 5 достаточно заметить, что искомые значения $\mu_{j} \sim e^{-\frac{\pi j}{\Omega}}$, при которых точка $P_{M}^{+}$ложится на устойчивое многообразие точки $R_{j}$, находятся при помощи формулы $(34)$, куда вместо $x$ и $\varphi$ следует подставить координаты точки $P_{M}^{+}$, соответственно, $\mu$ и $\varphi^{*}$. Так как $\rho<1$, то $\mu_{j} e^{\frac{\pi \rho j}{\Omega}}$ мало и аппроксимация (34) для $W_{j}^{s}$ действительно применима.

Для доказательства леммы 6 заметим, что так как неустойчивое многообразие $W_{j}^{u}$ имеет вид спирали, наматываюшейся на точку $P_{M}^{+}$, то вблизи $\mu_{j}$ найдутся значения $\mu$, при которых $W_{j}^{u}$ касается $W_{j}^{s}$. Из (34), (37) можно непосредственной проверкой убедиться, что касание квадратичное и что при изменении $\mu$ это касание расщепляется общим образом. Мы должны также проверить дополнительные условия невырожденности, необходимые для применения теоремы 2 из [16], а именно, что вблизи значений $\mu$, отвечающих гомоклиническому касанию, есть интервалы значений $\mu$, при которых соответствующее гиперболическое множество дикое.

Заметим, что неподвижная точка $R_{j}$ имеет (см. (33)) ровно один мультипликатор $\left(\nu_{2}\right)$, ближайший к единичной окружности изнутри (т.е. $R_{j}$ является $с е д л о м ~$ muna $(1,1)$ по терминологии $[16])$. При этом всего один мультипликатор лежит вне единичного круга. В этом случае условия невырожденности сводятся к двум 
требованиям: траектория гомоклинического касания не должнна лежсать в неведущем многообразии точки $R_{j}$, и всюду в точках данной траектории расширен-

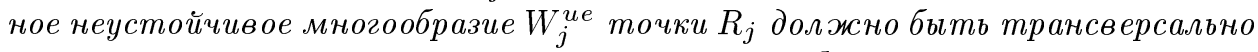
сильно устойчивому слоению устойчивого многообразия. Локально, неведушее многообразие $W_{j}^{s s}$ - это гладкое инвариантное многообразие, касательное в точке $R_{j}$ к собственному подпространству, отвечающему неведущим мультипликаторам $\nu_{3}, \ldots, \nu_{n}$. В нашем случае таковым как раз является слой слоения $\mathscr{N}^{s s} \cap \Pi$, проходящий через точку $R_{j}$, поэтому локально он совпадает с $W_{j}^{s s}$ (в силу единственности последнего). Известно, что неведушее многообразие неподвижной точки вкладывается в сильно устойчивое инвариантное слоение, причем, так как всюду на устойчивом многообразии это слоение определяется однозначно, то на устойчивом многообразии точки $R_{j}$ оно совпадает с $\mathscr{N}^{s s} \cap \Pi$.

Как мы отмечали, это слоение трансверсально трехмерному многообразию $\mathscr{M}_{\mu}$, которое содержит все траектории, целиком лежащие в малой окрестности гомоклинической петли седло-фокуса. В частности, $\mathscr{M}_{\mu} \cap \Pi$ содержит точку $R_{j}$ и рассматриваемую траекторию гомоклинического касания. В силу трансверсальности, $R_{j}$ - изолированная точка пересечения $W_{j}^{s s}$ и $\mathscr{M}_{\mu} \cap \Pi$, поэтому $W_{j}^{s s}$ не может содержать траекторий, асимптотических к $R_{j}$ и лежащих в $\mathscr{M}_{\mu} \cap \Pi$. Следовательно, рассматриваемая траектория гомоклинического касания действительно не лежит в $W_{j}^{s s}$.

Двумерное многообразие $\mathscr{M}_{\mu} \cap \Pi$ инвариантно и трансверсально $W_{j}^{s}$, и как следствие, оно касается в точке $R_{j}$ двумерного собственного подпространства, отвечающего мультипликаторам $\left(\nu_{1}, \nu_{2}\right)$. Любое инвариантное многообразие, касательное в неподвижной точке к собственной плоскости, отвечающей неустойчивому и ведущему устойчивому мультипликаторам, может рассматриваться как расширенное неустойчивое многообразие $W_{j}^{u e}$ : любое из них содержит неустойчивое многообразие и любые два из них касаются друг друга всюду на неустойчивом многообразии (детали см. в [21]). Так как $\mathscr{M}_{\mu} \cap$ П трансверсально слоению $\mathcal{N}^{s s} \cap \Pi$, то мы получаем, что действительно, в любой точке рассматриваемой негрубой гомоклинической траектории расширенное неустойчивое многообразие трансверсально сильно устойчивому слоению. Таким образом, условия невырожденности вьполнены и теорема 2 из [16] действительно применима в нашем случае.

Для завершения доказательства теоремы осталось доказать лемму 4. Представим полноторий П как полосу $\widetilde{\Pi}=D^{n-2} \prod \mathbb{R}^{1}$ (где $(x, z)$ служат координатами в $D^{n-2}$, а $\varphi-$ в $\left.\mathbb{R}^{1}\right)$, в которой точки $(x, z, \varphi)$ и $(x, z, \varphi+2 \pi k)$ отождествлены для любого целого $k$. Возьмем поднятие полнотория П на $\widetilde{\Pi}$, и пусть $\mathscr{P}$ - образ множества $T_{+}\left(\Pi_{+} \cup \Pi_{0}\right) \cup T_{-}\left(\Pi_{-} \cup \Pi_{0}\right)$ при данном поднятии. Так как $T_{+}\left(\Pi_{+} \cup \Pi_{0}\right) \cup T_{-}\left(\Pi_{-} \cup \Pi_{0}\right)$ стягиваемо в точку в П, то при всех $\mu \mathscr{P}$ лежит в ограниченной области $\widetilde{\Pi}$, т.е. для некоторого целого $s$

$$
\mathscr{P} \subset\left\{-\pi s<\varphi-\varphi^{*}<\pi s\right\}
$$

Так как для аттрактора $\mathscr{A}_{\mu}$ системы $X_{\mu}$ пересечение $\mathscr{A}_{\mu} \cap \Pi$ содержится в $T_{+}\left(\Pi_{+} \cup \Pi_{0}\right) \cup T_{-}\left(\Pi_{-} \cup \Pi_{0}\right)$, то поднятие в $\widetilde{\Pi}$ пересечения аттрактора с секущей содержится в $\mathscr{P}$, и следовательно, целиком лежит в ограниченной области $\left\{-\pi s<\varphi-\varphi^{*}<\pi s\right\}$.

Как было показано выше, устойчивое многообразие точки $R_{j}$ содержит кусок, задаваемый соотношениями $(34)-(36)$, где $\bar{k}$ достаточно велико и фиксировано, а $k$ 
растет пропорционально $j$ с ростом $j$ и стремлением $\mu$ к нулю. Мы можем считать, что $(k-\bar{k})$ делится на $2 s$ и что $(j-\bar{k}-s)$ четно, и нарезать поднятие $W_{j}^{s}$ на куски, отвечаюшие значениям $\left(\varphi-\varphi^{*}\right)$ из интервалов $(\pi \bar{k}-\pi j+2 \pi s l, \pi \bar{k}-\pi j+$ $2 \pi s(l+1))$, где $l=0, \ldots,(k-\bar{k}) /(2 s-1)$. Поскольку мы отождествляем точки, чья $\varphi$-координата отличается на кратное $2 \pi$, то можно сдвинуть кусок с номером $l$ на $-2 \pi(s l-(j-\bar{k}-s) / 2)$. В результате получим набор поверхностей $\mathscr{W}_{l}$ вида

$$
x=w_{l}(\varphi, z, \mu),
$$

где функции $w_{l}$ определены при $-\pi s<\varphi-\varphi^{*}<\pi s$ и

$$
w_{l} \sim e^{-\frac{\varphi-\varphi^{*}+\pi(\bar{k}+s)+2 \pi s l}{\Omega}}
$$

(см. (34)). В частности, для верхней из этих поверхностей, $w_{0}$, имеем

$$
x>\frac{1}{2} e^{-\frac{\pi \bar{k}+2 \pi s}{\Omega}}
$$

и для нижней поверхности, $w_{\bar{l}}$, где $\bar{l}=(k-\bar{k}) /(2 s-1)$,

$$
x<2 e^{-\frac{\pi k-2 \pi s}{\Omega}} .
$$

Очевидно, что ограничения (36) не мешают (поскольку $\rho<1$ ) выбрать $k$ и $\bar{k}$ так, чтобы поднятие точки $P_{M}^{+}$, чья $x$-координата равна $\mu$, лежало ниже поверхности $w_{0}$ и выше поверхности $w_{\bar{l}}$. Заметим, что на обеих этих поверхностях координата $x$ мала. Точка $P_{M}^{+}$принадлежит аттрактору $\mathscr{A}_{\mu}$ (так как это точка на сепаратрисе). Пусть $V$ - компонента связности множества $\mathscr{A}_{\mu} \cap \Pi$, содержащая точку $P_{M}^{+}$, и $\widetilde{V}$ - ее поднятие в $\widetilde{\Pi}$. Как отмечалось, множество $\widetilde{V}$ целиком лежит в области $\left\{-\pi s<\varphi-\varphi^{*}<\pi s\right\}$. По теореме $3 V$ содержит одну из точек $P_{1}^{+}, \ldots, P_{N}^{+}$или $P_{1}^{-}, \ldots, P_{N}^{-}$, где $N$ - число компонент связности множества $\mathscr{A}_{\mu} \cap \Pi$. Так как по предположению $M>N$, то точки $P_{i}^{+}, i=1, \ldots, N$, лежат на конечном расстоянии от $\Pi_{0}$ при всех малых $\mu$. Если при $\mu=0$ сепаратриса $\Gamma_{-}$не образует петли, то же самое справедливо и для точек $P_{i}^{-}, i=1, \ldots, N$. Таким образом, в этом случае компонента $V$ наряду с точкой $P_{M}^{+}$содержит точку, лежашую на конечном расстоянии от $\Pi_{0}$, т.е. координата $x$ этой точки отделена от нуля. Так как на поверхностях $w_{0}$ и $w_{\bar{l}}$ координата $x$ мала, то поднятие данной точки в $\widetilde{P}$ лежит вне области, ограниченной данными поверхностями (область $w_{\bar{l}}(\varphi, z, \mu)<x<$ $\left.w_{0}(\varphi, z, \mu)\right)$. В то же время, поднятие точки $P_{M}^{+}$лежит внутри этой области. Так как $\widetilde{V}$ связно, то это множество пересекает по крайней мере одну из поверхностей $w_{0}$ или $w_{\bar{l}}$.

Напомним, что данные поверхности представляют собой куски поднятия многообразия $W_{j}^{s}$ в $\widetilde{\Pi}$, т.е. мы получили, что при всех малых $\mu$ аттрактор содержит хотя бы одну точку на устойчивом многообразии траектории $L_{j}$. Отсюда следует, что он содержит саму траекторию $L_{j}$, ее неустойчивое многообразие и его замыкание. В частности, он содержит гиперболическое множество $\Lambda_{\mu}$.

Если же при $\mu=0$ сепаратриса $\Gamma_{-}$тоже образует петлю, то совершенно аналогично, при всех малых $\mu$ в окрестности этой петли лежит гиперболическое множество $\Lambda_{\mu}^{-}$. Для кусков устойчивого и неустойчивого многообразий однообходной 
периодической траектории из $\Lambda_{\mu}^{-}$справедливы представления вида $(34)$ и $(37)$ с переменой знака $x$ с положительного на отрицательный. Если $N^{+}<N<M-$ число из теоремы 3 , то точка $P_{N^{+}}^{+}$принадлежит компоненте $V$ множества $\mathscr{A}_{\mu} \cap \Pi$, пересекающей П 0 . Как мы отмечали, точка $P_{N^{+}}^{+}$лежит на конечном расстоянии от $\Pi_{0}$. Так как $V$ содержит и $P_{N^{+}}^{+}$, и некоторую точку на $\Pi_{0}$, то так же, как и выше, $V$ пересекает либо устойчивое многообразие одной из периодических точек из $\Lambda_{\mu}^{+}$ $\left(\right.$ если $\left.P_{N^{+}}^{+} \in \Pi_{+}\right)$, либо одной из периодических точек из $\Lambda_{\mu}^{-}\left(\right.$если $\left.P_{N^{+}}^{+} \in \Pi_{-}\right)$. Соответственно, либо $\Lambda_{\mu}^{+}$, либо $\Lambda_{\mu}^{-}$лежит в аттракторе. Но так как неустойчивые многообразия периодических траекторий из $\Lambda_{\mu}^{-}$пересекают трансверсально $\Pi_{0}$ (см. (37)), а устойчивые многообразия периодических точек из $\Lambda_{\mu}^{+}$подходят сколь угодно близко к П 0 при достаточно малых $\mu$ (см. (34)-(36)), то получаем, что неустойчивое многообразие $\Lambda_{\mu}^{-}$пересекает устойчивое многообразие $\Lambda_{\mu}^{+}$, т.е. в любом случае $\Lambda_{\mu}^{+}$входит в аттрактор, что завершает доказательство теоремы.

Мы указали области в пространстве динамических систем, в которых аттрактор содержит дикое множество вместе с его неустойчивым многообразием. В этих областях плотны системы, для которых одна из периодических траекторий дикого множества имеет траекторию гомоклинического касания устойчивого и неустойчивого многообразий. Согласно [4] в любой окрестности такой системы есть системы, у которых устойчивое и неустойчивое многообразия этой периодической траектории имеют касания произвольно высокого порядка. Так как гомоклинические траектории принадлежат неустойчивому многообразию и, следовательно, входят в аттрактор, мы получаем следующий результат.

ТЕОРема 5. B построенных областях плотны системы, аттрактор которых содержит негрубые гомоклинические траектории произвольно высоких порядков касания.

Данная теорема показывает, что бифуркации рассматриваемого аттрактора не могут быть полностью описаны ни в каком конечно-параметрическом семействе. Другим отражением этого свойства является то, что в аттрактор могут входить сколь угодно вырожденные периодические траектории.

Теорема 6. B построенных областях плотны системы, аттрактор которых содержит негрубые периодические траектории, имеющие один мультипликатор, равный единице, и произвольно большое число равных нулю ляпуновских величин.

Эта теорема также является следствием плотности в построенных областях систем с гомоклиническими касаниями: согласно [4] в любой окрестности системы с гомоклиническим касанием есть системы со сколь угодно вырожденньми периодическими траекториями. Следующая лемма уточняет результат из [4] (мы не воспроизводим здесь доказательство, чтобы не загромождать изложение).

ЛЕмма 7. Пусть некоторая система имеет седловую периодическую траекторию $L$ с мультипликаторами $\nu_{1}, \nu_{2}, \ldots, \nu_{n}$. Предположим, что $\left|\nu_{1}\right|>1$ u $1>\left|\nu_{2}\right|>\left|\nu_{m}\right|, m=3, \ldots, n$ (это означает, в частности, что $\nu_{1} u \nu_{2}$ вещественнь). Пусть также

$$
\left|\nu_{1} \cdot \nu_{2}\right|>1
$$


Предположим, что неустойчивое и устойчивое многообразия $L$ имеют касание порядка $k$ по некоторой траектории. Тогда в произвольном $k$-параметрическом семействе общего положения, содержащем данную систему, найдутся значения параметров, отвечающие существованию негрубой периодической траектории с одним мультипликатором, равным единице, одним мультипликатором, больиим единиць по абсолютной величине, и остальными мультипликаторами внутри единичного круга, и у ограничения отображсеня Пуанкаре на иентральное многообразие (отвечающее единичному мультипликатору) первые $(k-1)$ ляпуновских величин равнь нулю. При этом устойчивое многообразие данной периодической траектории имеет траектории трансверсального пересечения с неустойчивым многообразиe.M $L$.

Чтобы применить данную лемму к доказательству теоремы 6, заметим, что седловые периодические траектории с негрубыми гомоклиническими траекториями произвольных порядков касания входят в аттрактор согласно теореме 5 (для плотного множества систем из рассматриваемых областей). Как видно из (33), данные седловые периодические траектории (траектории $L_{j}$ ) удовлетворяют условиям леммы 7. Следовательно, в указанных областях плотны системы, для которых неустойчивое многообразие седловой периодической траектории в аттракторе пересекает устойчивое многообразие какой-либо негрубой периодической траектории сколь угодно высокого наперед заданного порядка вырождения. Так как эта траектория принадлежит замыканию неустойчивого многообразия траектории из аттрактора, то она сама принадлежит аттрактору.

Применив лемму 7 к однопараметрическому $(k=1)$ семейству $X_{\mu}$ из теоремы 4 , мы получим, что в интервалах $\Delta_{i}$ плотны значения $\mu$, при которых аттрактор содержит негрубую периодическую траекторию типа седло-седло. У такой траектории один мультипликатор больше единицы по абсолютной величине, один мультипликатор равен единице и соответствующая первая ляпуновская величина не равна нулю, и остальные мультипликаторы лежат внутри единичного круга. Неустойчивое многообразие такой траектории трехмерно. Так как каждая траектория входит в аттрактор вместе со своим неустойчивым многообразием, мы получаем следующий результат.

ТЕОРема 7. В интервалах $\Delta_{i}$ плотны значения $\mu$, при которых топологическая размерность аттрактора $\mathscr{A}_{\mu}$ равна трем.

\section{Список литературы}

1. Newhouse S. E. The abundance of wild hyperbolic sets and non-smooth stable sets for diffeomorphisms // Inst. Hautes Études Sci. Publ. Math. 1979. V. 50. P. 101-151.

2. Newhouse S. E. Diffeomorphisms with infinitely many sinks // Topology. 1974. V. 13. P. 9-18.

3. Gonchenko S. V., Shil'nikov L.P., Turaev D. V. On models with non-rough Poincare homoclinic curves // Phys. D. 1993. V. 62. № 1-4. P. 1-14.

4. Гонченко С. В., Тураев Д. В., Шильников Л. П. Динамические явления в многомерных системах с негрубой гомоклинической кривой Пуанкаре // Докл. АН. 1993. Т. 330. № 2. C. $144-147$.

5. Gonchenko S. V., Shil'nikov L.P., Turaev D. V. Dynamical phenomena in systems with structurally unstable Poincaré homoclinic orbits // Interdisc. J. Nonlinear Sci. CHAOS. 1996. V. 6. № 1. P. 1-17. 
6. Афраймович В. С., Быков В.В., ШІильников Л.П. О возникновении и структуре аттракторов Лоренца // Докл. АН СССР. 1977. Т. 234. № 2. С. 336-339.

7. Афраймович В. С., Быков В. В., Шильников Л. П. О притягивающих негрубых предельных множествах типа аттрактора Лоренца // Труды ММО. 1982. Т. 44. С. 150-212.

8. Овсянников И.М., Шильников Л. П. О системах с гомоклинической кривой седло-фокуса // Матем. сб. 1986. Т. 130 (172). №4. С. 552-570.

9. Hirsh M.W., Pugh C. C., Shub M. Invariant manifolds // Lect. Notes in Math. V. 583, 1977.

10. Guckenheimer J., Williams R.-F. Structural stability of Lorenz attractors // Astérisque. 1979. V. 50. P. 307-320.

11. Аносов Д. В. Геодезические потоки на замкнутых римановых многообразиях отрицательной кривизны // Труды МИАН. 1967. Т. 90.

12. Песин $\mathcal{A}$. Б. Характеристические показатели Ляпунова и гладкая эргодическая теория // УМН. 1977. Т. 32. № 4. С. 55-111.

13. Аносов Д. В., Арансон С. Х., Бронштейн И. У., Гринес В. З. Гладкие динамические системы. II // Итоги науки и техн. Совр. пробл. матем. Фундам. напр. Т. 1. М.: ВИНИТИ, 1985. C. $151-242$.

14. Auslender J. Generalized recurrence in dynamical systems // Contributions to differential equations. V. 3. New York: John Wiley and Sons, Inc., 1964. P. 65-74.

15. Шильников Л. П. К вопросу о структуре расширенной окрестности грубого состояния равновесия типа седло-фокус // Матем. сб. 1970. Т. 81 (123). №1. С. 92-113.

16. Гонченко С. В., Тураев Д. В., Шильников Л. П. О существовании областей Ньюхауса вблизи систем с негрубой гомоклинической кривой Пуанкаре (многомерный случай) // Докл. АН. 1993. Т. 329. № 4. С. 404-407.

17. Palis J., Viana $M$. High-dimension diffeomorphisms displaying infinitely many periodic attractors // Ann. of Math. 1994. V. 140. P. 207-250.

18. Hayashi $S$. On the solution of $C^{1}$-stability conjecture for flow // Preprint.

19. Feroe J. A. Homoclinic orbits in a parametrized saddle-focus system // Phys. D. 1993. V. 62. № 1-4. P. 254-262.

20. Шильников Л. П. Об одной задаче Пуанкаре-Биркгофа // Матем. сб. 1967. Т. 74 (116). № 4. C. 378-397.

21. Turaev D. V. On dimension of non-local bifurcational problems // Int J. Bifur. Chaos. Appl. Sci. Engrg. 1996. V. 6. № 5. P. 919-948.

22. Sandstede B. Center manifolds for homoclinic solutions // Preprint, 1994.

Weierstrass Institute of Mathematics;

НИИ прикладной математики и кибернетики,

Нижний Новгород

E-mail: shilnikov@focus.nnov.su
Поступила в редакцию 20.01.1997 